\title{
Heart rate, arterial distensibility, and optimal performance of the arterial tree
}

\author{
Carla Silva ${ }^{\mathrm{a}, \mathrm{b}}, \mathrm{A}$. Heitor Reis ${ }^{\mathrm{b}, \mathrm{c}, *}$ \\ a Polytechnic Institute of Tomar, Qta. do Contador, 2300-313 Tomar, Portugal \\ ${ }^{\mathrm{b}}$ Évora Geophysics Centre, R. Romão Ramalho, 59, 7002-554 Évora, Portugal \\ c Physics Department, University of Évora, R. RomãoRamalho, 59, 7002-554 Évora, Portugal
}

\section{A R T I C L E I N F O}

\section{Article history:}

Accepted 22 July 2014

\section{Keywords:}

Pulsatile flow

Heart rate

Arterial distensibility

\begin{abstract}
A B S T R A C T
In this study we explore the ability of a previously developed model of pulsatile flow for explaining the observed reduction of arterial distensibility with heart rate. The parameters relevant for the analysis are arterial wall distensibility together with permeability and reflection coefficients of the end capillaries. A non-specific artery and the ensemble of tissues supplied by that artery were considered in the model. The blood current within that artery was equalized to the sum of all micro currents in the tissues supplied by that artery. A formula emerged that relates changes in arterial distensibility with heart rate, and also with some particular aspects of microcirculation. Then, that formula was tested with data of distensibilities of the radial and carotid arteries observed at the heart rates of 63, 90, and 110 b.p.m. The formula correctly predicted the trend of decreased distensibility with heart rate for both arteries. Moreover, due to the fact that the carotid artery supplies the brain, and because the Blood-Brain barrier is highly restrictive to colloids in the blood, for the carotid artery the formula predicted a less marked decrease in distensibility than in the case of the radial artery feeding muscle tissue, which has a greater permeability to colloids, a trend that was confirmed by data. It was found that reduction of arterial distensibility with heart rate was greater in arteries that supply end capillaries with high permeability and low reflection coefficients.
\end{abstract}

(c) 2014 Elsevier Ltd. All rights reserved.

\section{Introduction}

It has been long recognized that pulsatile blood flow performs the best than continuous flow because it induces both lower total peripheral resistance and mean arterial pressure (Mavroudis, 1978), and also better blood perfusion (Taylor et al., 1982). The distensibility coefficient of the vessel wall is defined as $\beta=(2 / D)(d D / d P)$ where $P$ is pressure within the vessel, and $D$ is vessel diameter. Henceforth the term distensibility is used to mean distensibility coefficient.

On the other hand, a recent model of pulsatile flow predicts that if distensibility is kept constant, arterial impedance must decrease with pulse frequency (Silva and Reis, 2014). Actually, if pulse frequency (heart rate) and therefore the blood current increases in response to needs of organs in the body, then it makes sense that arterial impedance is reduced to ease the access of blood. On the other hand, if arterial impedance decreases with distensibility (Silva and Reis,

\footnotetext{
* Corresponding author at: Physics Department, University of Évora, R. Romão Ramalho, 59, 7002-554, Évora, Portugal. Tel.: + 351266745 372; fax: +351266745 394 .

E-mail addresses: carlasilva@ipt.pt (C. Silva), ahr@uevora.pt (A.H. Reis).
}

2014) one would expect that increased blood current would lead to decreased arterial impedance with heart rate.

However, many studies have shown that, in humans, arterial distensibility varies inversely with heart rate (Asmar et al., 1996; Liang et al., 1999; Giannattasio et al., 2003; Pitcher et al., 2010; Koskinen et al., 2011; Chrysohoou et al., 2013). The same effect has been observed in rats (Mircoli et al., 1999). Though, according to the model above referred (Silva and Reis, 2014) increased heart rate leads to lower arterial impedance, the observed increased arterial stiffness with heart rate actually increases impedance. Apparently, this behavior does not make sense because easing blood flow is sought to be the objective of the circulatory system.

An extended search in the pertinent literature also revealed some studies that concluded that arterial stiffness was not affected by heart rate (Wilkinson et al., 2002; Aizawa et al., 2009). However, these two studies have some particularities: in (Wilkinson et al., 2002) arterial stiffness changes with heart rate were indirectly estimated through the augmentation index calculated from the blood pressure waveform, while in (Aizawa et al., 2009) arterial stiffness was "determined before and 10 min after graded arm-cycling exercise".

On the other hand, some other studies (Kingwell et al., 1997; Currie et al., 2009; Jae-Bin et al., 2013) found that whole body arterial 


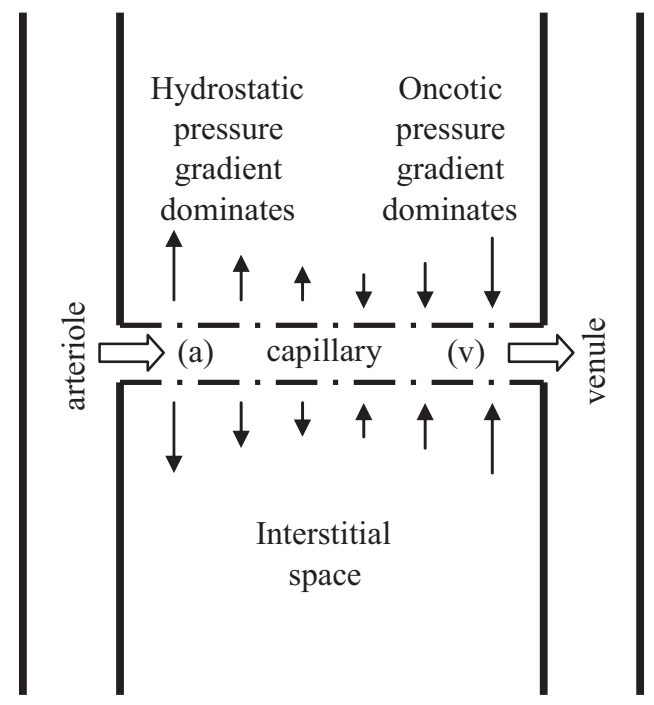

Fig. 1. Microcirculation: blood enters the capillary at the arteriolar end (a), water, salts and colloids are driven into the interstitial space by the capillary gradient, and return into the capillary driven by the oncotic gradient at the venular end (v).

compliance (WBAC) and then arterial distensibility was increased after cycling and treadmill exercise with relation to the values prior to exercise. This is a different result that compares arterial distensibility before and after the period in which heart rate is increased.

The decrease of arterial distensibility with heart rate is somewhat counter-intuitive, and challenges the current paradigm of human physiology. From the physiological point of view, no explanation has yet been presented. In the following we offer an explanation based on the assumption of optimal hemodynamic performance of the arterial tree, and show that increase in arterial stiffness with heart rate may be understood as the adjustment of the arterial tree on the way for global optimization of its performance. For this purpose we first take a closer look to microcirculation in the capillaries forming the end of the arterial tree.

\section{Microcirculation and Starling forces}

Blood is transported downstream in the arterial tree until it reaches the end capillaries that bridge arteriole and venule ends, which deliver it to the interstitial fluid that bathes every cell (see Fig. 1). Capillaries have opening of various widths according to the tissue to which blood is supplied. Many capillaries may turn impermeable to the bigger colloids in the blood, namely the proteins, therefore regulating their delivery to the interstitial fluid. Special classes of proteins called albumins constitute about $50 \%$ of human plasma protein and are very important as carriers of hydrophobic substances (e.g. lipid soluble hormones, bile salts, free fatty acids) (Farrugia, 2010). Water and other small molecules are generally free to pass through capillary openings.

In this way, filtration occurs along the capillary driven by the difference in hydrostatic pressure $\Delta P_{c i}=P_{c}-P_{i}$ between the capillary $\left(P_{c}\right)$ and the interstitial space $\left(P_{i}\right)$, therefore increasing the concentration of colloids that are not allowed to pass into the interstitial fluid. As the result a colloid osmotic pressure - oncotic pressure difference $\Delta \Pi_{c i}=\Pi_{c}-\Pi_{i}-$ develops between the interstitial fluid and the blood within the capillaries, which opposes the pressure gradient that drives the blood from the capillaries to the interstitial space (i.e. the space between cells that is bathed by the interstitial fluid). Within the capillary, hydrostatic pressure decreases from $P_{a}$ (at the end of the arteriole) to $P_{v}$ (at the beginning of the venule), $\Delta P_{c}=P_{a}-P_{v}$.

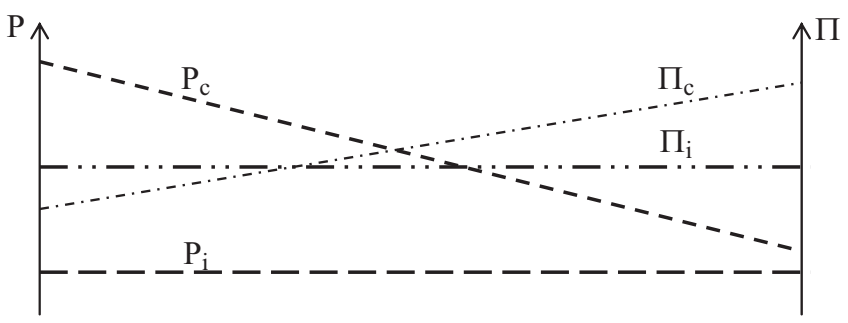

(a)

Fig. 2. Variation of capillary hydrostatic pressure $\left(P_{c}\right)$, interstitial hydrostatic pressure $\left(P_{i}\right)$, capillary oncotic pressure $\left(\Pi_{c}\right)$, and interstitial oncotic pressure $\left(\Pi_{i}\right)$, between arteriolar $(\mathrm{a})$ and venular $(\mathrm{v})$ ends of the capillary. $\Pi_{c}$ increases within the capillary due to loss of fluid to the interstitial space. $P_{i}$ and $\Pi_{i}$ are constant in the interstitial space (Taylor, 1981; Seifter et al., 2005).

Hence, the blood leaving the end of the arteriole splits into two currents: one of them flows within the interstitial space; another one flows within the capillary. Both currents merge together at the entrance of the venula.

The current flowing into the interstitial space may be described by Starling's equation (Taylor, 1981; Seifter et al., 2005)

$i_{c i}=K_{c i} \Delta P_{c i}-\sigma_{c i} \Delta \Pi_{c i}$

where $i_{c i}$ is the net current between the capillary and the interstitial space, $K_{c i}$ and $\sigma_{c i}$ respectively stand for filtration coefficient and reflection coefficient of the capillary section in which $i_{c i}$ exists, $\Pi_{c}$ and $\Pi_{i}$ represent oncotic pressure of the colloids in the capillary and the interstitial space, respectively. Though in the literature, $K_{c i}$ and $\sigma_{c i}$ are termed "coefficients", in fact they represent conductances that are proportional to the extension of the capillary in which exchange of fluids occur. The reflection coefficient is null for a capillary wall permeable to all colloids in the blood. The first term in the r.h.s of Eq. (1) represents the current from the capillary to the interstitial space while the second one stands for the current from the interstitial space onto the capillary that is driven by oncotic pressure gradient. At the arteriolar end the hydrostatic driven current dominates, hence there is a net influx to the interstitial space, while the opposite occurs at the venular end where a net outflow towards the capillary (see Fig. 2).

The current within the capillary is driven by the hydrostatic pressure difference $\Delta P_{c}=P_{a}-P_{v}$ and is given by:

$i_{c}=\Delta P_{c} / Z_{c}$

where $Z_{c}$ stands for capillary impedance. In this way, the total current leaving the arteriolar end is given by

$i=i_{i}+i_{c}=K_{c i} \Delta P_{c i}-\sigma_{c i} \Delta \Pi_{c i}+\Delta P_{c} / Z_{c}$

On the other hand, the total current entering the venular end is composed of the current from the interstitial space into the capillary:

$i_{v}=i_{i c}+i_{c}=K_{i c}\left(\Delta P_{c}-\Delta P_{c i}\right)+\sigma_{i c} \Delta \Pi_{c i}+\Delta P_{c} / Z_{c}$

where $K_{i c} \neq K_{c i}$ and $\sigma_{i c} \neq \sigma_{c i}$ respectively stand for filtration coefficient and reflection coefficient of the capillary section in which $i_{c i}$ exists. Note that here we have considered the general case in which $K_{c i} \neq K_{i c}$ and $\sigma_{c i} \neq \sigma_{i c}$. Additionally, due to mass conservation for the steady state one has

$i=i_{v}+i_{\text {lymph }}$

where $i_{\text {lymph }}$ stands for the rate at which lymph is drained from the interstitial space to the lymphatic circulation. Therefore, from Eqs. (3)-(5) one obtains

$i_{\text {lymph }}=\left(K_{i c}+K_{c i}\right) \Delta P_{c i}-K_{i c} \Delta P_{c}-\left(\sigma_{c i}+\sigma_{i c}\right) \Delta \Pi_{c i}$ 


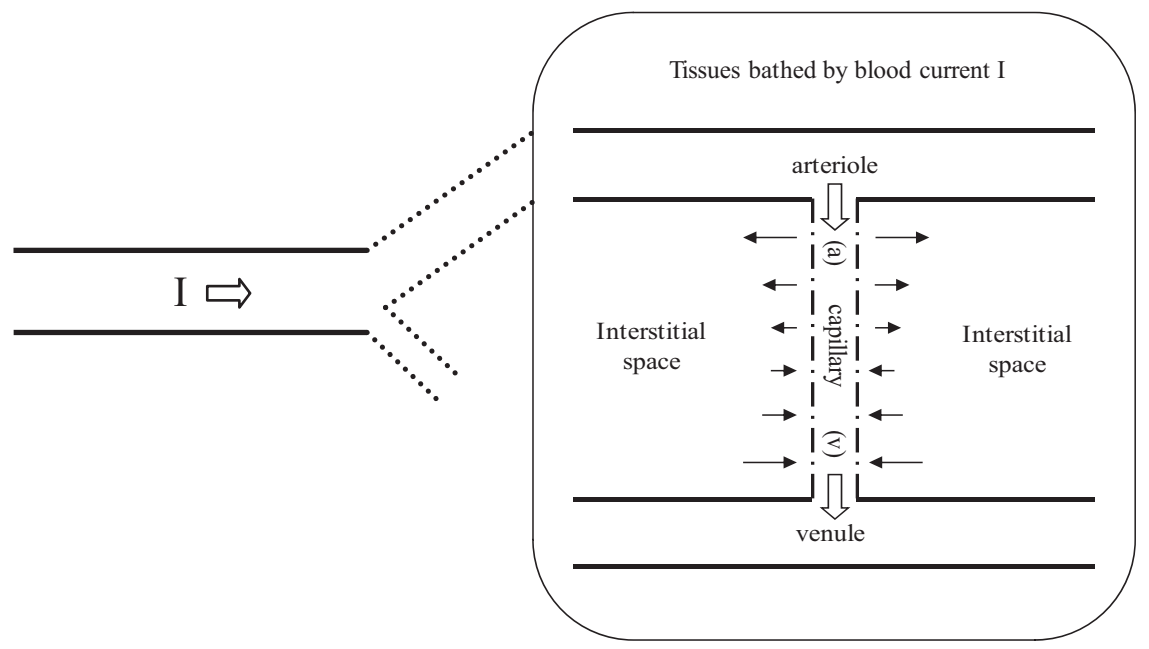

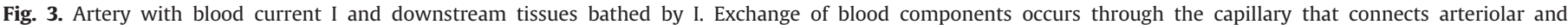
venular ends.

In the tissues that do not possess lymphatic circulation (e.g. brain, eyes) special overflow drainage systems allow for the removal of excess fluid (Taylor, 1981).

\section{Heart rate and optimal performance of the arterial tree}

Let us consider an artery that feeds blood to the tissues downstream (Fig. 3). In an artery of diameter $D$ and length $L$, (aspect ratio $x=L / D$ ), with pulsatile flow of frequency $\omega$, under pressure difference $\Delta P$ the average current $I$ reads (see Silva and Reis, 2014)

$I=\Delta P / Z, \quad$ with $Z=k_{A} x y^{-1}\left(1+\omega^{2} / \hat{\omega}^{2}\right)^{-1 / 2}$

where $k_{A}=128 \mu \pi^{-1}, \mu$ is dynamic viscosity of the fluid, $y=D^{3}$, $k=k_{A} \pi \beta / 4, \beta=(2 / D)(d D / d P)$ is the distensibility coefficient of the vessel wall, $P$ is pressure, and $\hat{\omega}=1 / k x^{2}$ represents the characteristic radial frequency of the vessel.

The blood current $I$ in that artery equals the sum of the blood currents leaving every venular end in the tissues feed by that artery

$I=\sum_{n} i_{n}$

or, according to Eq. (3):

$I=\sum_{n}\left(K_{c i} \Delta P_{c i}-\sigma_{c i} \Delta \Pi_{c i}+\Delta P_{c} / Z_{c}\right)_{n}$

The pressure drop along the capillary is proportional the absolute value of pressure drop along the artery, i.e. $\Delta P_{c}=\theta \Delta P$. For an optimally performing tree $\theta=1$. In such a tree the same pressure drop occurs at level of branching (see Reis, 2006). In this way, by using Eqs. (5)-(9) one obtains

$Z^{-1}=\sum_{n}\left(\left[a_{k}\left(\sigma_{c i}+\sigma_{i c}\right)-\sigma_{c i}\right] \frac{\Delta \Pi_{c i}}{\Delta P}+a_{k} \frac{i_{l y m p h}}{\Delta P}+\theta\left(a_{k} K_{i c}+Z_{c}^{-1}\right)\right)_{n}$

where $a_{k}=K_{c i} /\left(K_{c i}+K_{i c}\right)$. Besides we note that $Z_{c} \simeq R_{c}$ because the flow in capillaries is almost steady because in there the pressure wave is very much attenuated. As a consequence, the variation of the impedance with pulse rate is negligible, i.e. $\partial Z_{c} / \partial \omega \simeq 0$.

By taking the derivate of both members of Eq. (9) with respect to pulse frequency $\omega$ one obtains

$\frac{\nu}{\beta} \frac{\partial \beta}{\partial \nu} \simeq-1-\gamma$ with

$$
\begin{aligned}
\gamma= & \sum_{n}\left(\left\{\left[a_{k}\left(\sigma_{c i}+\sigma_{i c}\right)-\sigma_{c i}\right] \frac{\Delta \Pi}{\Delta P}+a_{k} \frac{i_{l y m p h}}{\Delta P}\right\} \frac{\hat{\nu}}{\nu}\left(1+\frac{\hat{\nu}^{2}}{\nu^{2}}\right)^{1 / 2} \frac{R \nu}{\Delta P} \frac{\partial(\Delta P)}{\partial \nu}\right)_{n} \\
& +\left(1+\frac{\hat{\nu}^{2}}{\nu^{2}}\right) \beta \frac{\nu \partial P}{\partial \nu}
\end{aligned}
$$

where $\beta$ is distensibility coefficient, $\nu=\omega / 2 \pi, \hat{\nu}=\hat{\omega} / 2 \pi$ stand for normal heart rate and characteristic frequency, respectively, and $R=k_{A} x y^{-1}$ stands for flow resistance of the artery (see Eq. (7)). The last term in the r.h.s of Eq. (11a) depends on $\hat{\nu}^{2} / \nu^{2}$, which may be significant in some arteries (e.g. the carotid, see next Section 4.), and also on $\partial P / \partial \nu$. However, the data in (Giannattasio et al., 2003) do not show a clear trend with respect to changes in arterial pressure with heart rate, and more likely show that $\partial P / \partial \nu \sim 0$, therefore indicating that the last term in the r.h.s of Eq. (11a) may be neglected. In this way, $\gamma$ may be considered as approximately constant, what allows Eq. (11) to be integrated by using its average value $\langle\gamma\rangle$. Then, by integrating both members of Eq. (11) with respect to $\nu$ one finds:

$\beta=\beta_{0}\left(\nu / \nu_{0}\right)^{-1-\langle\gamma\rangle}$

where $\beta_{0}$ and $\nu_{0}$ are reference values. Because $\partial(\Delta P) / \partial \nu>0,\langle\gamma\rangle$ is generally positive. On the other hand, for arteries that supply tissues without lymphatic vessels $i_{\text {lymph }}=0$, and also tissues exist for which the reflection coefficient $\sigma$ is very high, while permeability $K_{c i}$ (and then $a_{k}$ ) approaches zero. This is the case of the brain for which $a_{k} \approx 0$, and $\sigma_{c i}$ is very high, and therefore a less pronounced decrease in arterial distensibility with heart rate is expected to occur with the carotid as compared with the arteries that supply tissues with small values of reflection coefficient (e.g. the skeletal muscle).

\section{The cases of the carotid and radial arteries}

Very few data of arterial distensibility at various heart rates are found in the literature. To our knowledge only Giannattasio and co-workers (Giannattasio et al., 2003) published data of distensibilities of the carotid and radial arteries at the heart rates of 63, 90 and 110 b.p.m (beats per minute). In the following, we will analyze those data in the light of Eq. (12).

As regards the value of $\langle\gamma\rangle$ respecting the carotid and radial arteries we observe that the first one supplies the brain whose 
Table 1

Distensibilities at various heart rates from (Giannattasio et al., 2003) and parameter $\langle\gamma\rangle$ (see. Eq. (12)).

\begin{tabular}{lll}
\hline Heart rate (b.p.m.) & \multicolumn{2}{l}{ Distensibility $\left(1 /(\mathrm{mm} \mathrm{Hg}) \times 10^{-3}\right)$} \\
\cline { 2 - 3 } & radial artery & carotid artery \\
\hline 63 & 0,0908 & 0184 \\
90 & 0,0474 & 0,149 \\
110 & 0,0395 & 0,120 \\
$\langle\gamma\rangle$ & $\mathbf{0 . 5 3 1}$ & $\mathbf{- 0 . 2 5 4}$ \\
\hline
\end{tabular}

tissues have a very high refection coefficient due to the highly selective barrier that separates the circulating blood from the extracellular fluid in the brain. Conversely, the radial artery that supplies the skeletal muscle in the arm presents a much lower reflection coefficient. On the other hand, by using the definition of characteristic radial frequency $\hat{\omega}=1 / k x^{2}$ (see Eq. (7)), together with the pertinent values taken from (Reymond et al., 2009) it is found that the characteristic frequencies of the carotid and radial arteries, $\hat{\nu}=\hat{\omega} / 2 \pi$, are 3020 and 736 b.p.m., respectively. As a consequence the ratio $\hat{\nu} / \nu$ corresponding to the carotid artery is about 40 times larger than that of the radial artery.

By using the data of (Giannattasio et al., 2003) and fitting Eq. (12) to a logarithmic scale: $\ln \left(\beta / \beta_{0}\right) \simeq-(1+\langle\gamma\rangle) \ln \left(\nu / \nu_{0}\right)$, one obtains the values of $\langle\gamma\rangle$ shown on Table 1 .

These results deserve scrutiny in the light of Eq. (11a).

\subsection{Radial artery}

The radial artery supplies the skeletal muscle in the arm, which has high permeability $K_{c i}\left(a_{k} \approx 0.5\right)$, and very low reflection coefficient, $\sigma_{c i}$, therefore from Eq. (11a) we conclude that $\langle\gamma\rangle$ must be positive. Moreover $i_{\text {lymph }}$ may be significant in the arm thus adding a positive contribution to the already positive value of $\langle\gamma\rangle$. The value $\langle\gamma\rangle=0.531$ found for the radial artery from patient data (Giannattasio et al., 2003) is in accordance with the tendency anticipated from Eq. (11a).

\subsection{Carotid artery}

This artery supplies the brain, whose capillaries due to the Blood-Brain Barrier have very low permeability $K_{c i}$, (and then $a_{k} \approx 0$ ) together with a very high reflection coefficient $\sigma_{c i}$. On the other hand, as referred above, though small drainage might occur by a special system (Taylor, 1981), the brain has no lymphatic vessels, then $i_{\text {lymph }}=0$. By taking into account all these aspects we conclude from Eq. (11a) that $\langle\gamma\rangle$ must be negative. In addition, the ratio $\hat{\nu}^{2} / \nu^{2}$ is high therefore modulating the value of $\langle\gamma\rangle$, accordingly. By using data from (Giannattasio et al., 2003) we found $\langle\gamma\rangle=-0.254$, which due to the properties of the radial artery is also in accordance with Eqs. (11) and (12).

In this way, the reduction of arterial distensibility with heart rate appears as the result of the adjustment of the artery that supplies some part of the body to the particular features of the microcirculatory tree (end capillaries) and the interstitial space in that part of the body. Mircoli and co-workers (Mircoli et al., 1999) found that in rats "in predominantly elastic-type arteries, the stiffening effect of tachycardia is exerted independently of sympathetic modulation". In fact, and in line with this finding no external control by any system external to the arterial tree (e.g. the sympathetic nervous system) was invoked in the analysis above developed, rather the effect of arterial stiffening with heart rate sprang of the continuity of blood flow (Eq. (8)) together with the coefficients that characterize the exchanges between the end capillaries and the interstitial space (Eq. (9)).

\section{Conclusions}

In this paper, we showed that the effect of reduction of arterial distensibility with heart rate may be understood based on the physical properties of the arterial tree that supplies some tissues and the particular properties of blood exchanges between the end capillaries and the interstitial space in those tissues. The parameters that are relevant for the analysis are arterial wall distensibility, permeability and reflection coefficients of the end capillaries, which together account for arterial vasoactivity.

It was found that reduction of arterial distensibility with heart rate was greater in arteries that supply blood to end capillaries with high permeability coefficients together with low reflection coefficients. This trend was confirmed through the use of data of distensibilities at the heart rates of 63, 90 and 110 b.p.m. respecting the radial and carotid arteries.

In line with findings that showed that the effect of arterial stiffening with heart rate was virtually independent of sympathetic modulation, the present results showed that the assumption of continuity of blood flow together with the coefficients that characterize the exchanges between the end capillaries and the interstitial space may be sufficient to explain the observed effects.

\section{Conflict of interest statement}

The authors declare no conflict of interest.

\section{Acknowledgment}

The authors acknowledges the funding provided by the CGE, under the contract with FCT the (Portuguese Science and Technology Foundation), Pest/OE/CTE/UI0078/2014.

\section{Appendix A. Supporting information}

Supplementary data associated with this article can be found in the online version at http://dx.doi.org/10.1016/j.jbiomech.2014.07.025.

\section{References}

Aizawa, K., Mendelsohn, M.E., Overend, T.J., Petrella, R.J., 2009. Effect of upper body aerobic exercise on arterial stiffness in older adults. J. Aging Phys. Act. 17, $468-478$.

Asmar, R., Scuteri, A., Topouchian, J., Brisac, A.M., Maldonado, J., Cloarec, L., Safar, M., 1996. Arterial distensibility and circadian blood pressure variability. Blood Press. Monit. 1 (4), 333-338.

Chrysohoou, C., Metaxa, V., Skoumas, J., Lagoudakou, S., Athanassopoulou, S., Kosyfa, H., Oikonomou, E., Felekos, J., Tsiachris, D., Masoura, C., Pitsavos, C., Stefanadis, C., 2013. Aortic artery distensibility shows inverse correlation with heart rate variability in elderly non-hypertensive, cardiovascular disease-free individuals: the Ikaria Study. Heart Vessel. 28, 467-472.

Currie, K.D., Thomas, S.G., Goodman, J.M., 2009. Effects of short-term endurance exercise training on vascular function in young males. Eur. J. Appl. Physiol. 107, 211-218.

Farrugia, A., 2010. Albumin usage in clinical medicine: tradition or therapeutic? Transfus. Med. Rev. 24 (1), 53-63.

Giannattasio, C., Vincenti, A., Failla, M., Capra, A., Cirò, A., De Ceglia, S., Gentile, G., Brambilla, R., Mancia, G., 2003. Effects of heart rate changes on arterial distensibility in humans. Hypertension 42, 253-256.

Jae-Bin, S., Woo-Young, C., Sang-Hyun, K., Myung-A, K., Joo-Hee, Z., 2013. Immediate impact of exercise on arterial stiffness in Humans. World J. Cardiovascu. Dis. 3, 40-45.

Kingwell, B.A., Jennings, G.L., Berry, K.L., Dart, A.M., Cameron, J.D., 1997. Arterial compliance increases after moderate-intensity cycling. Am. J. Physiol.-Heart Circ. Physiol. 42, 2186-2191.

Koskinen, T., Juonala, M., Kähönen, M., Jula, A., Keltikangas-Järvinen, L., Viikari, J., Välimäki, I., Laitinen, T., Raitakari, O.T., 2011. Relations between carotid artery distensibility and heart rate variability:the cardiovascular risk in young finns study. Auton. Neurosci.: Basic Clinic. 161, 75-80. 
Liang, Y.L., Gatzka, C.D., Du, X.J., Cameron, J.D., Kingwell, B.A., Dart, A.M., 1999. Effects of heart rate on arterial compliance in men. Clin. Exp. Pharmacol. Physiol. 26, 342-346.

Mavroudis, C., 1978. To pulse or not to pulse. Ann. Thorac. Surg. 25, 259-271.

Mircoli, L., Mangoni, A.A., Giannattasio, C., Mancia, G., Ferrari, A.U., 1999. Heart ratedependent stiffening of large arteries in intact and sympathectomized rats. Hypertension 34, 598-602.

Pitcher, A., Leeson, P., Forfar, C., Trevitt, C., Francis, J.M., Neubauer, S., Petersen, S.E., 2010. Aortic distensibility decreases during exercise in normal volunteers. J. Cardiovasc. Magn. Reson. 12 (1), 136.

Reis, A.H., 2006. Constructal Theory: from engineering to physics, and how flow systems develop shape and structure. Appl. Mech. Rev. 59 (5), 269-282.

Reymond, P., Merenda, F., Perren, F., Rüfenacht, D., Stergiopulos, N., 2009. Validation of a one-dimensional model of the systemic arterial tree. Am. J. Physiol.-Heart Circ. Physiol. 297, H208-H222.
Seifter, J., Sloane, D., Ratner, A., 2005. Concepts in Medical Physiology. Lippincott Williams, \& Wilkins, Baltimore p. 162.

Silva, C., Reis, A.H., 2014. Structure and adaptation of arteries to pulsatile flow - The case of the ascending aorta. Med. Phys. 41, 063701.

Taylor, A.E., 1981. Capillary fluid filtration. Starling forces and lymph flow. Circ. Res. 49 (3), 557-575.

Taylor, K.M., Bain, W.H., Davidson, K.G., Turner, M.A., 1982. Comparative clinical study of pulsatile and non-pulsatile perfusion in 350 consecutive patients. Thorax 37, 324-330.

Wilkinson, I.B., Mohammad, N.H., Tyrrell, S., Hall, I.R., Webb, D.J., Paul, V.E., Levy, T., Cockcroft, J.R., 2002. Heart rate dependency of pulse pressure amplification and arterial stiffness. Am. J. Hypertens. 15, 24-30. 